\title{
Surface Properties and Compatibility with Blood of New Quaternized Polysulfones
}

\author{
Raluca Marinica Albu, Ecaterina Avram, Iuliana Stoica, Emil Ghiocel Ioanid, \\ Dumitru Popovici, Silvia Ioan
}

Institute of Macromolecular Chemistry, Iasi, Romania.

E-mail: ioan_silvia@yahoo.com

Received January $13^{\text {th }}$, 2011; revised January $16^{\text {th }}$, 2011; accepted January $21^{\text {st }}, 2011$.

\begin{abstract}
The paper describes some properties of new quaternized polysulfones obtained by quaternization of chloromethylated polysulfone with different tertiary amines- $N, N$-dimethylethylamine and $N, N$-dimethyloctylamine. Hydrophilic/hydrophobic properties, morphological aspects and interface properties with red blood cells and platelets are affected by the alkyl radicals and by history of the formed films from solutions in $N, N$-dimethylformamide/methanol and $N, N$-dimethylformamide/water solvent/nonsolvent mixtures. The results obtained are useful in biomedical applications, including evaluation of bacterial adhesion to the surfaces, or utilization of modified polysulfones as semipermeable membranes.
\end{abstract}

Keywords: Quaternized Polysulfones, Surface Properties, Blood Compatibility

\section{Introduction}

In recent decades, considerable attention has been devoted to the investigation of new applications of polysulfones, and also, of chloromethylated and quaternized polysulfones, which was mainly due to their specific properties. Literature showed that polysulfones and their derivatives were widely used as new functional materials in biochemical, industrial and medical fields, due to their structure and physical characteristics, such as good optical properties, high thermal and chemical stability, mechanical strength, resistance to extreme $\mathrm{pH}$ values and low creep [1-4]. Chain rigidity is derived from the relatively inflexible and immobile phenyl and $\mathrm{SO}_{2}$ groups, while toughness - from the connecting ether oxygen [4].

The polysulfone can be modified to improve its performance for specific applications [4,5], by chloromethylation, a reaction of considerable interest from both theoretical and practical points of view, such as obtaining of precursors for functional membranes, coatings, ion exchange resins, ion exchange fibers and selectively permeable membranes [6,7]. Also, quaternization with ammonium groups is an efficient method to obtain some properties recommended in various applications, e.g. as biomaterials and semipermeable membranes. These groups can modify hydrophilicity (of special interest for biomedical applications) [8], the antimicrobial action $[9,10]$, solubility characteristics $[11,12]$, allowing water permeability and separation [13,14]. In addition, the functional groups are an intrinsic requirement for affinity, ion exchange and other specialy membranes [15]. Moreover, the bioapplication of polysulfones can be divided in two categories, namely blood contacting devices - for example, hemodialysis, hemodiafiltration and hemofiltration as membrane, and cell or tissue contacting devices for example, bioreactor made by hollow fibre membrane, nerve generation through polysulfone semipermeable hollow membrane, etc [5].

In previous publications, the synthesis [16-18] and some solution properties [11,19-23] of modified polysulfones have been presented. Studies have been carried out on the quaternization reaction of chloromethylated polysulfones, for obtaining water soluble polymers with various amounts of ionic chlorine. The conformational behavior in solution and the experimental and theoretical results on the preferential adsorption coefficients versus solvent composition have been discussed in correlation with the interaction parameters of quaternized polysulfones/mixed solvents $[10,20,23]$. Surface wettability and hydrophilicity trends, as well as the morphological characteristics of some modified polysulfones were also analyzed, for biomaterials and semipermeable membrane applications $[10,12,22,24]$. On the other hand, it is wellknown that surface-induced blood coagulation is one of the main problems in the development of blood-contacting materials. From a clinical point of view, literature 
shows that a biomaterial can be considered as hemocompatible only when its interaction with blood does not provoke damage of blood cells or change in the structure of plasma proteins [25-27]. The surface free energy of biomaterials and the corresponding values of the work of spreading can be used as characterization parameters for predicting cell spreading onto their surfaces and hence, for establishing their blood compatibility.

The objective of the present study was to investigate the morphological characteristics of quaternized polysulfones obtained from chloromethylated polysulfones with tertiary amines, N,N-dimethylethylamine and $\mathrm{N}$, $\mathrm{N}$-dimethyl-octylamine. The corresponding films, obtained from solutions in N,N-dimethylformamide (DMF) /methanol $(\mathrm{MeOH})$ and DMF/water mixtures, were analyzed by atomic force microscopy, to emphasize the influence of casting solutions on the morphological properties. The results were correlated with the hydrophilic/ hydrophobic properties and red blood cells and platelets compatibility. Influence of the alkyl radical sizes from the side groups was evidenced comparatively with the modified polysulfones with $\mathrm{N}$-dimethyloc-tylammonium chloride pendant groups [27]. In this context, such investigations were discussed in correlation with the computerized chemical structure, which provides a generalized view on the chemical conformations of the repeating units, realized by the HyperChem professional program (Demo version). This representation helps to identify aspects of molecular structure which may be relevant to the structure-property problem here under consideration, blood compatibility included.

\section{Materials and Methods}

\subsection{Materials}

UDEL-3500 polysulfone (PSF) (Union Carbide, $\mathrm{M}_{\mathrm{n}}=$ 39000; $M_{w} / M_{n}=1.625$ ), a commercial product, was purified by repeated reprecipitations from chloroform and dried for $24 \mathrm{~h}$ in vacuum, at $40^{\circ} \mathrm{C}$, before being used in the synthesis of chloromethylated polysulfone. A mixture of commercial paraformaldehyde with an equimolar amount of chlorotrimethylsilane $\left(\mathrm{Me}_{3} \mathrm{SiCl}\right)$ as a chloromethylation agent, and stannic tetrachloride $\left(\mathrm{SnCl}_{4}\right)$ as a catalyst, was used for the chloromethylation reaction of polysulfone, at $50^{\circ} \mathrm{C}$. The reaction time necessary to obtain chloromethylated polysulfones with $6.58 \%$ chlorine content (CMPSF) was 72 h [7]. Finally, the samples were dried under vacuum at $40^{\circ} \mathrm{C}$.

Polysulfones with different alkyl side groups, PSFDMEA and PSF-DMOA, were synthesized by reacting chloromethylated polysulfone with different tertiary amines-N,N-dimethylethylamine (DMEA) and N, Ndimethyloctylamine (DMOA), respectively. The quarternization reaction was performed in DMF, using a CMPSF/ tertiary amine molar ratio of $1: 1.5$, for $24 \mathrm{~h}$. The quaternary polymers were isolated from the reaction medium by precipitation in diethylether, washed three times with diethylether, and dried for $48 \mathrm{~h}$ under vacuum, at room temperature. The contents of ionic chlorine of 2.89 and 3.23, and total chlorine of 3.10 and 3.29 for PSF- DMEA and PSF-DMOA, respectively, were determined by Schoninger's method followed by potentiometric titration with $\mathrm{AgNO}_{3}$, using an automatic TitraLab Radiometer 840.

The ratios between ionic chlorine and the total chlorine contents show that the quaternization reaction of CMPSF occurs at a transformation degree close to $98 \%$. Thus, one may consider that almost all chloromethylenic groups were quaternized. Scheme 1 presents the general chemical structures and conformational structures - obtained by a computerized method using the HyperChem professional program (Demo version) considering four structural units, of PSF-DMEA and PSF-DMOA.

\subsection{Contact Angle}

Contact angle analysis for surface tension investigations and atomic force microscopy (AFM) measurements were realized on quaternized polysulfones films. PSF-DMEA and PSF-DMOA were dissolved in DMF, DMF/MeOH (over the 75/25\% - 25/75\% v/v and 75/25\% - 45/55\% v/v composition range, respectively), and DMF/Water (over the $75 / 25 \%$ - $40 / 60 \% \mathrm{v} / \mathrm{v}$ and $75 / 25 \%$ - 50/50\% v/v composition range, respectively), to reach the concentrations of approximatively $7 \mathrm{~g} / \mathrm{dL}$. The solutions were cast on a glass plate and initially solidified by slow drying in saturated atmosphere with the used solvent, and finally by drying at $50^{\circ} \mathrm{C}$ under vacuum. Uniform drops of the $2 \mu \mathrm{L}$ test liquid were deposited on the film surface and the contact angles were measured after $30 \mathrm{~s}$, with a video-based optical contact angle measuring device, in a temperature of $25^{\circ} \mathrm{C}$. The used test liquids are water, methylene iodide $\left(\mathrm{CH}_{2} \mathrm{I}_{2}\right)$, and 1-brom-naphtalin (1-Bn).

\subsection{Atomic Force Microscopy}

Atomic force microscopy (AFM) measurements were performed in air, at room temperature $\left(23^{\circ} \mathrm{C}\right)$, in the tapping mode using a Scanning Probe Microscope (Solver PRO-M, NT-MDT, Russia) with commercially available NSGI0 cantilevers. The manufacture's value for the probe tip radius was $10 \mathrm{~nm}$ and for the typical force constant was $11.5 \mathrm{~N} / \mathrm{m}$. In the tapping mode, the cantilever was oscillated at a frequency of $286 \mathrm{kHz}$, over a $20 \times 20 \mu \mathrm{m}^{2}$ scan area for each sample.

\section{Results and Discussion}

\subsection{Surface Tension Parameters}

The geometric mean method (GM) (Equations (1) and (2)) 

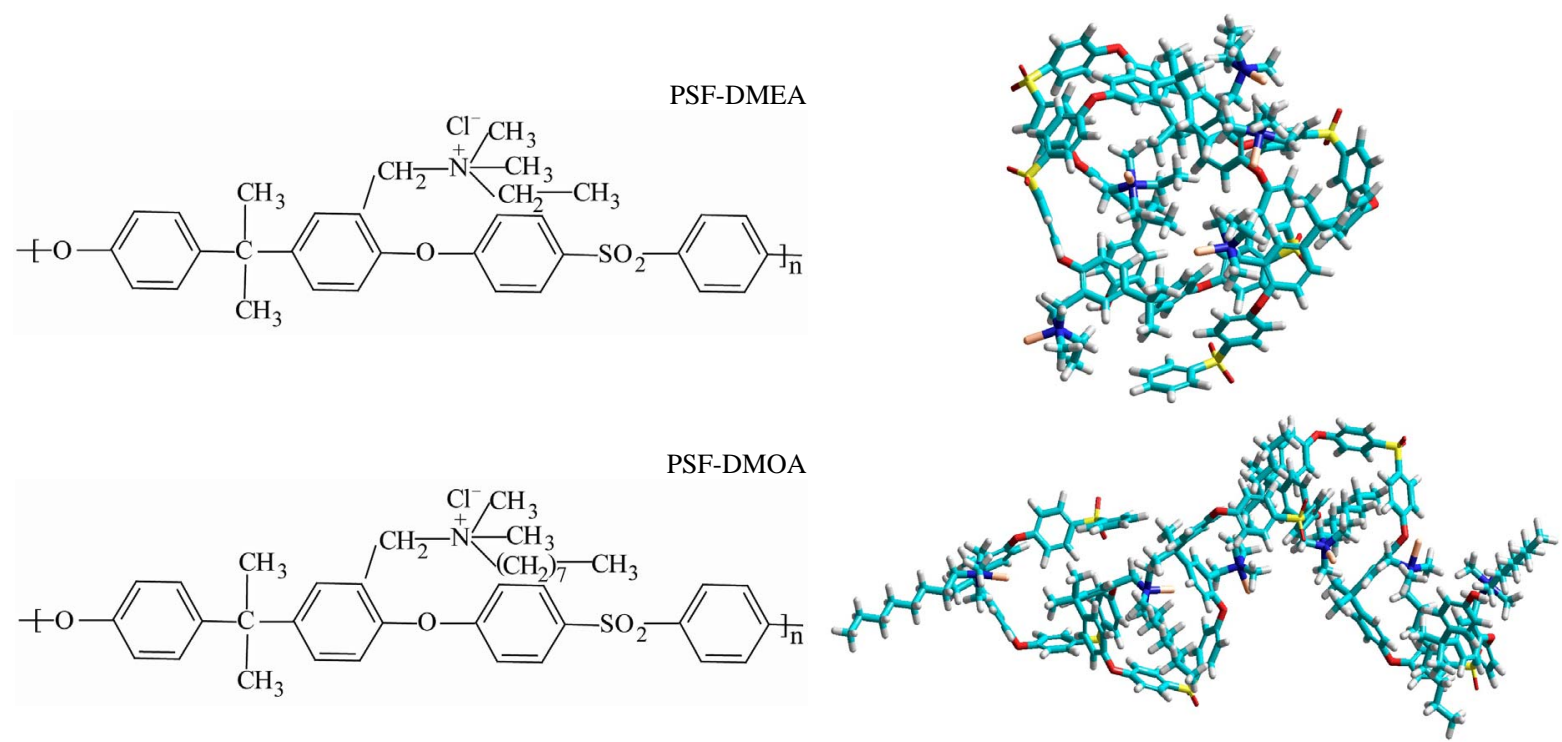

Scheme 1. Chemical structures and conformational structures with minimized energies, considering four repeating units of polysulfones with quaternary groups.

$[28,29]$ and the acid/base method (LW/AB) (equation (3) and (4) $[30,31]$ were utilized for calculating the surface tension parameters of PSF-DMEA and PSF-DMOA, with surface properties of test liquids [32] from Table 1, and the contact angles measured between these solvents and quaternized polysulfone films from Table 2. The contact angles between these solvents and PSF-DMOA films are presented in previous paper [27].

$$
\begin{gathered}
\frac{1+\cos \theta}{2} \cdot \frac{\gamma_{l v}}{\sqrt{\gamma_{l v}^{d}}}=\sqrt{\gamma_{s v}^{p}} \cdot \sqrt{\frac{\gamma_{l v}^{p}}{\gamma_{l v}^{d}}}+\sqrt{\gamma_{s v}^{d}} \\
\gamma_{s v}=\gamma_{s v}^{d}+\gamma_{s v}^{p}
\end{gathered}
$$

where $\theta$ is the contact angle determined for test liquids, subscripts " $l v$ " and " $s v$ " denote the liquid-vapor and surface-vapor interfacial tension, respectively, while superscripts " $p$ " and " $d$ " denote the polar and disperse components, respectively, of total surface tension, $\gamma_{s v}$.

$$
\begin{gathered}
1+\cos \theta=\frac{2}{\gamma_{l v}} \cdot\left(\sqrt{\gamma_{s v}^{L W} \cdot \gamma_{l v}^{L W}}+\sqrt{\gamma_{s v}^{+} \cdot \gamma_{l v}^{-}}+\sqrt{\gamma_{s v}^{-} \cdot \gamma_{l v}^{+}}\right) \\
\gamma_{s v}^{L W / A B}=\gamma_{s v}^{L W}+\gamma_{s v}^{A B}
\end{gathered}
$$

where $\gamma_{s v}^{A B}=2 \cdot \sqrt{\gamma_{s v}^{+} \cdot \gamma_{s v}^{-}}$, superscript “ $L W / A B$ ” indicates the total surface tension, and also, superscript " $A B$ " and " $L W$ " represent the polar component obtained from the electron-donor, $\gamma_{s v}^{-}$, and the electron-acceptor, $\gamma_{s v}^{+}$, interactions, and the disperse component, respectively.

Table 3 shows the results for the surface tension components, evaluated with both methods. The surface tension parameters are influenced by the solvent/nonsolvent composition from which the films had been prepared.
Some studies have reported that the chain shape of a polymer in solution could affect the morphology of the polymer in bulk. In this context, the conformations of both PSF-DMEA and PSF-DMOA are affected by the charged groups from different alkyl radicals of the studied quaternized samples, and also by the compositition of the solvent mixtures. Thus, Figure 1 depicts the variation of intrinsic viscosity with the volume fraction of DMF, in $\mathrm{DMF} / \mathrm{MeOH}$ and DMF/water mixtures, for PSF-DMEA

\begin{tabular}{|c|c|c|c|c|c|}
\hline Liquid & $\gamma_{l v}$ & $\gamma_{l v}^{d}$ & $\gamma_{l v}^{p}$ & $\gamma_{l v}^{+}$ & $\gamma_{l v}^{-}$ \\
\hline Water [32] & 72.80 & 21.80 & 51.00 & 25.50 & 25.50 \\
\hline $\begin{array}{l}\text { Methylene iodide } \\
\left(\mathrm{CH}_{2} \mathrm{I}_{2}\right)[32]\end{array}$ & 50.80 & 50.80 & 0 & 0.72 & 0 \\
\hline $\begin{array}{l}\text { 1-Brom-naphtalin } \\
\text { (1-Bn) [32] }\end{array}$ & 44.40 & 44.40 & 0 & 0 & 0 \\
\hline Red blood cell [35] & 36.56 & 35.20 & 1.36 & 0.01 & 46.2 \\
\hline Platelet [35] & 118.24 & 99.14 & 19.10 & 12.26 & 7.44 \\
\hline
\end{tabular}

Table 1. Surface tension parameters $(\mathrm{mN} / \mathrm{m})$ of the liquids used for contact angle measurements, red blood cells and platelets.

Table 2. Contact angle $\left({ }^{\circ}\right)$ of different liquids on films prepared from solutions of PSF-DMEA in DMF/MeOH and DMF/water (\% v/v) (column 1).

\begin{tabular}{cccc}
\hline Solvent mixtures & W & Contact angle \\
& 71 & 28 & 17 \\
\hline 100/0 DMF/MeOH & 70 & 31 & 22 \\
75/25 DMF/MeOH & 61 & 30 & 20 \\
50/50 DMF/MeOH & 63 & 31 & 24 \\
25/75 DMF/MeOH & 59 & 35 & 21 \\
75/25 DMF/water & 60 & 33 & 18 \\
50/50 DMF/water & 56 & 33 & 16 \\
40/60 DMF/water & & &
\end{tabular}


Table 3. Surface tension parameters $(\mathrm{mN} / \mathrm{m})$ and contribution of the polar component to the total surface tension $(\%)$ for quaternized polysulfone films PSF-DMEA and PSF-DMOA prepared from solutions in DMF/MeOH and DMF/water (v/v \%), according to the geometrical mean method and the acid/base method (equations (1) - (4)).

\begin{tabular}{|c|c|c|c|c|c|c|c|c|c|c|}
\hline \multirow{2}{*}{ Solvent mixtures ${ }^{-}$} & \multicolumn{4}{|c|}{ Geometrical mean method } & \multicolumn{6}{|c|}{ Acid/base method } \\
\hline & $\gamma_{s v}^{d}$ & $\gamma_{s v}^{p}$ & $\gamma_{s v}$ & $\gamma_{s v}^{p} / \gamma_{s v}$ & $\gamma_{s v}^{L W}$ & $\gamma_{s v}^{+}$ & $\gamma_{s v}^{-}$ & $\gamma_{s v}^{A B}$ & $\gamma_{s v}^{L W / A B}$ & $\gamma_{s v}^{A B} / \gamma_{s v}^{L W / A B}$ \\
\hline \multicolumn{11}{|c|}{ PSF-DMEA, DMF/MeOH } \\
\hline $100 / 0$ & 43.7 & 5.9 & 49.7 & 11.9 & 42.5 & 3.6 & 2.6 & 6.2 & 48.7 & 12.6 \\
\hline $75 / 25$ & 42.5 & 6.6 & 49.2 & 13.5 & 41.3 & 4.3 & 2.8 & 6.9 & 48.1 & 14.3 \\
\hline $50 / 50$ & 42.9 & 10.7 & 53.7 & 20.0 & 41.8 & 9.9 & 2.5 & 9.9 & 51.7 & 19.2 \\
\hline $25 / 75$ & 42.1 & 10.0 & 52.1 & 19.1 & 40.6 & 6.5 & 4.1 & 10.4 & 51.0 & 20.4 \\
\hline \multicolumn{11}{|c|}{ PSF-DMEA, DMF/water } \\
\hline $75 / 25$ & 41.8 & 12.2 & 54.0 & 22.7 & 41.5 & 21.4 & 0.1 & 3.18 & 44.68 & 7.1 \\
\hline $50 / 50$ & 42.6 & 11.4 & 54.0 & 21.1 & 42.3 & 19.0 & 0.2 & 3.83 & 46.08 & 8.3 \\
\hline $40 / 60$ & 42.8 & 13.4 & 56.3 & 23.9 & 42.7 & 25.4 & 0.2 & 4.15 & 46.85 & 8.9 \\
\hline \multicolumn{11}{|c|}{ PSF-DMOA, DMF/MeOH [27] } \\
\hline $100 / 0$ & 40.9 & 5.1 & 46.0 & 11.0 & 42.4 & 0.4 & 1.4 & 1.5 & 43.9 & 3.4 \\
\hline $75 / 25$ & 39.6 & 3.0 & 42.6 & 6.9 & 42.7 & 0.2 & 0.8 & 0.8 & 43.5 & 1.8 \\
\hline $50 / 50$ & 43.6 & 1.9 & 45.2 & 4.1 & 43.1 & 1.5 & 2.0 & 1.8 & 44.9 & 3.9 \\
\hline $45 / 55$ & 39.1 & 3.6 & 42.7 & 8.5 & 41.3 & 1.0 & 0.5 & 1.4 & 42.7 & 3.3 \\
\hline \multicolumn{11}{|c|}{ PSF-DMOA, DMF/water [27] } \\
\hline $75 / 25$ & 38.4 & 7.5 & 46.0 & 16.4 & 41.4 & 0.5 & 1.2 & 1.5 & 42.9 & 3.5 \\
\hline 60.40 & 38.5 & 8.6 & 47.1 & 18.3 & 40.2 & 0.8 & 3.5 & 3.3 & 43.6 & 7.6 \\
\hline $50 / 50$ & 41.4 & 5.9 & 47.4 & 12.5 & 42.8 & 0.5 & 1.3 & 1.6 & 44.4 & 3.6 \\
\hline
\end{tabular}

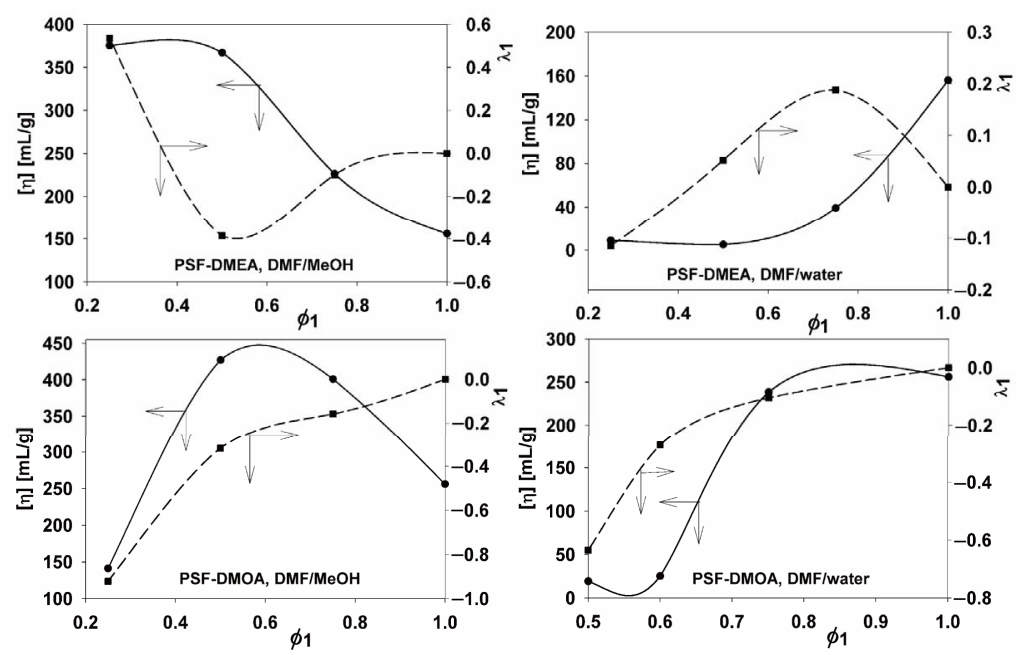

Figure 1. Influence of DMF volume fraction, $\phi_{1}$, on intrinsic viscosity, $[\eta]$, and on the experimental values of the preferential adsorption coefficient, $\lambda_{1}$, in DMF/MeOH and DMF/water, at $25^{\circ} \mathrm{C}$, for PSF-DMEA and PSF-DMOA samples.

and PSF-DMOA, respectively [23]. For the PSF-DMEA in the $\mathrm{DMF} / \mathrm{MeOH}$ system, one may observe that the polymer coil dimension decreases with increasing the DMF content in the 0.25 - 1 volume fraction domain; below a 0.25 volume fraction of DMF, the polymer pre- cipitates. For the same polymer, but in a DMF/water solvent mixture, the dimensions increase with increasing the DMF content, starting from approximately the same volume fraction of DMF. The PSF-DMOA coil dimesions possess maximum values in $\mathrm{DMF} / \mathrm{MeOH}$ and $\mathrm{DMF} /$ 
water, around 0.6 and 0.8 DMF volume fractions, respectively. For volume fractions of DMF below 0.25 in $\mathrm{DMF} / \mathrm{MeOH}$ and 0.5 in DMF/water, the PSF-DMOA precipitates due to the nature of the alkyl radicals and content of nonsolvent in the system. Also, the values of intrinsic viscosity are higher for PSF-DMOA-with bulky carbon atoms in the alkyl side chain, compared with PSF-DMEA, where the alkyl side chain possesses two carbon atoms. Therefore, for a given composition of the $\mathrm{DMF} / \mathrm{MeOH}$ and DMF/water solvent mixtures, one of the components is preferentially adsorbed by the quaternized polysulfone molecules in the direction of a thermodynamically most effective mixture.

These aspects influence the surface properties of the polymer. Moreover, according to previous data [27], it was found out that PSF evidences the lowest hydrophilicity, induced by the aromatic rings connected by one carbon and two methyl groups, oxygen elements, and sulfonic groups, while chloromethylation of PSF with the functional group $-\mathrm{CH}_{2} \mathrm{Cl}$ increases hydrophilicity (see the values of surface tension for PSF and CMPSF in Table 3 from reference [27]). On the other hand, PSF-DMEA films possess low polar surface tension parameters, but slightly higher than those for PSF-DMOA. The hydrophobic character is given by the ethyl radical from the $\mathrm{N}$-dimethylethylammonium chloride pendant group and by the octyl radical from the $\mathrm{N}$-dimethyloctylammonium chloride pendant group, respectively, as visualized in the conformational structures from Scheme 1. Furthermore, the electron donor interactions, $\gamma_{s v}^{-}$, are smaller than the electron acceptor ones, $\gamma_{s v}^{+}$, for PSF-DMEA, and electron donor interactions, $\gamma_{s v}^{-}$, exceed the electron acceptor interactions, $\gamma_{s v}^{+}$, for PSF-DMOA, caused by the inductive phenomena from alkyl radical. The results reflect the capacity of the N-dimethylethylammonium chloride or $\mathrm{N}$-dimethyloctylammonium chloride pendant groups to determine the acceptor or donor character of the polar terms, generated by these inductive phenomena.

\subsection{Surface and Interfacial Free Energy}

The effect of alkyl radicals of quaternized polysulfones and of the history of the films formed from solutions on surface properties was analyzed by surface free energy, $\Delta G_{w}$ - expressing the balance between surface hydrophobicity and hydrophilicity (equation (5)) [32], by interfacial free energy between two particles of quaternized polysulfones in water phase, $\Delta G_{s w s}^{G M}$ (equations (6) and (7)), and by the work of spreading of water, $W_{s}$ (equation (8)).

$$
\Delta G_{w}=-\gamma_{l v} \cdot\left(1+\cos \theta_{\text {water }}\right)
$$

where $\gamma_{l v}$ and $\theta_{\text {water }}$ are given in Tables 1 and 2, respectively.

$$
\begin{gathered}
\Delta G_{s w s}^{G M}=-2 \cdot \gamma_{s l} \\
\gamma_{s l}=\left(\sqrt{\gamma_{l v}^{p}}-\sqrt{\gamma_{s v}^{p}}\right)^{2}+\left(\sqrt{\gamma_{l v}^{d}}-\sqrt{\gamma_{s v}^{d}}\right)^{2} \\
W_{s}=W_{a}-W_{c} \\
=2 \cdot\left[\left(\gamma_{s v}^{L W} \cdot \gamma_{l v}^{d}\right)^{1 / 2}+\left(\gamma_{s v}^{+} \cdot \gamma_{l v}^{-}\right)^{1 / 2}+\left(\gamma_{s v}^{-} \cdot \gamma_{l v}^{+}\right)^{1 / 2}\right]-2 \cdot \gamma_{l v}
\end{gathered}
$$

According to literature [33,34] which specifies that $\Delta G_{w}>-113 \mathrm{~mJ} / \mathrm{m}^{2}$ for more hydrophobic materials, $\Delta G_{w}$ evidences a high hydrophobicity for both samples, depending on the conditions of films preparation (Table 4). Moreover, the interfacial free energy, $\Delta G_{s w s}^{G M}$ evaluated from solid-liquid interfacial tension, $\gamma_{s l}$, (equation (7)) has negative values (Table 4). Therefore, an attraction occurs between the two polymer surfaces, $s$, immersed in water, $w$, confirming the hydrophobic characteristics of both polymers, with higher hydrophobicity for PSF-DMOA films. Also, the hydrophobicity of these polymers was described by the work of spreading of water, $W_{s}$, over the surface, which represents the difference between the work of water adhesion, $W_{a}$, and the work of water cohesion, $W_{c}$. According to the negative values of the interfacial free energy of PSF-DMEA and PSF-DMOA, the work of spreading of water, $W_{s, w}$, (Figure 2) takes negative values, caused by the hydrophobic surfaces, where the work of water adhesion is low, comparatively with the work of cohesion; at the same time, it is observed that $W_{s, w, P S F-D M E A}>W_{s, w, P S F-D M O A}$.

\subsection{Blood-Quaternized Polysulfone Interactions}

Blood compatibility is dictated by the manner in which their surfaces interact with blood constituents, like red blood cells and platelets. To analyze the possibilities of using the polysulfone with $\mathrm{N}$-dimethylethylammonium and N-dimethyloctylammonium chloride groups in biomedical applications, and for establishing its compatibility with blood, equation (8) was used, where $W_{s, r b c}$ and $W_{s, p}$ describe the work of spreading of red blood cells and platelets [35]; when blood is exposed to a biomaterial surface, adhesion of cells occurs and the extent of adhesion decides the life of the implanted biomaterials; thus, cellular adhesion to biomaterial surfaces could activate coagulation and the immunological cascades. Therefore, cellular adhesion has a direct bearing on the thrombogenicity and immunogenicity of a biomaterial, and thus dictates its blood compatibility. In this paper, we used the work of adhesion of the red blood cells as a parameter for characterizing biomaterials versus cell adhesion. The materials which exhibit a lower work of adhesion would lead to a lower extent of cell adhesion than those with a higher work of adhesion. 


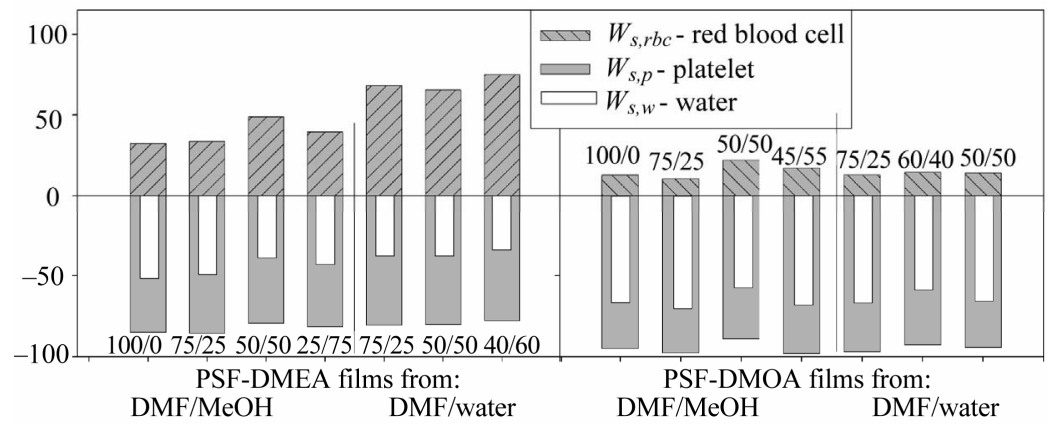

Figure 2. Work of spreading of water, of red blood cells and of platelets over the surface of PSF-DMEA and PSF-DMOA films prepared in different DMF/MeOH and DMF/water solvent mixture.

Table 4. Water interfacial tensions $\left(\gamma_{s l}\right)$ and surface free energy $\left(\Delta G_{w}\right)$ for PSF-DMEA and PSF-DMOA films prepared in different DMF/MeOH and DMF/water $(\% \mathrm{v} / \mathrm{v})$, and interfacial free energy $\left(\Delta G_{s w s}^{G M}\right)$ between two particles of quaternized polysulfones in water phase.

\begin{tabular}{ccccccc}
\hline & \multicolumn{3}{c}{$\gamma_{s l}$} & \multicolumn{2}{c}{$\Delta G_{w}$} & \multicolumn{2}{c}{$\Delta G_{\text {sws }}^{\text {GM }}$} \\
\cline { 2 - 7 } Solvent mixtures & \multicolumn{3}{c}{ DMF/MeOH } & \\
\cline { 2 - 7 } & PSF-DMEA & PSF-DMOA [27] & PSF-DMEA & PSF-DMOA [27] & PSF-DMEA & PSF-DMOA [27] \\
\cline { 2 - 7 } $100 / 0$ & 25.95 & 26.92 & -67 & -95.30 & -1.90 & -53.84 \\
$75 / 25$ & 24.26 & 32.06 & -68 & -87.94 & -48.52 & --64.12 \\
$50 / 50$ & 18.47 & 37.15 & -75 & -111.38 & -36.94 & -74.30 \\
$45 / 55$ & - & 29.91 & - & -89.18 & - & -59.82 \\
$25 / 75$ & 19.15 & - & -74 & - & -38.30 & - \\
& & & & & & - \\
$75 / 25$ & 16.50 & 21.66 & -77 & -102.41 & -32.30 & -43.32 \\
$60 / 40$ & - & 20.08 & - & -103.57 & - & -40.16 \\
$50 / 50$ & 17.60 & 25.30 & -76 & -97.70 & -35.20 & -50.60 \\
$40 / 60$ & 15.57 & - & -79 & - & -31.14 & - \\
\hline
\end{tabular}

Considering the surface energy parameters $\left(\gamma_{l v}, \gamma_{l v}^{d}\right.$, $\gamma_{l v}^{+}, \gamma_{l v}^{-}$) given in Table 1 for red blood cells and platelets, the work of spreading of blood cells and platelets was estimated by equation (8), with surface free parameters for films prepared from $\mathrm{DMF} / \mathrm{MeOH}$ and $\mathrm{DMF} /$ water solutions listed in Table 4.

Figure 2 shows positive values for the work of spreading of red blood cells, $W_{s, r b c}$, and negative values for the work of spreading of platelets, $W_{s, p}$, suggesting a higher work of adhesion comparatively with that of cohesion for the red blood cells, but a smaller work of adhesion comparatively with the one of cohesion for platelets. These results suggest that the exposure of platelets to PSF-DMEA and PSF-DMOA films determines an increase of platelets cohesion, which is higher for PSFDMOA films, and that a good hydrophobicity can be correlated with a good adhesion of the red blood cells on the surface of the polysulfone films.

In summary, both red blood cells and platelets are extremely important in deciding the blood compatibility of a material. Moreover, it is known that adhesion of the red blood cells onto a surface, e.g. modified polysulfones, requires knowledge of the interactions with the vascular components. Thus, endothelial glycocalyx along with the mucopolysaccharides adsorbed to the endothelial surface of the vascular endothelium reject clotting factors and platelets-which have a significant role in thrombus formation [36]. In this context, adhesion of the red blood cells and cohesion of platelets to surface films must be discussed in correlation with future specific biomedical applications. These results seem to be applicable for evaluating bacterial adhesion to the surfaces, and could be subsequently employed for studying possible implanted induced infections, or for obtaining semipermeable membranes. 

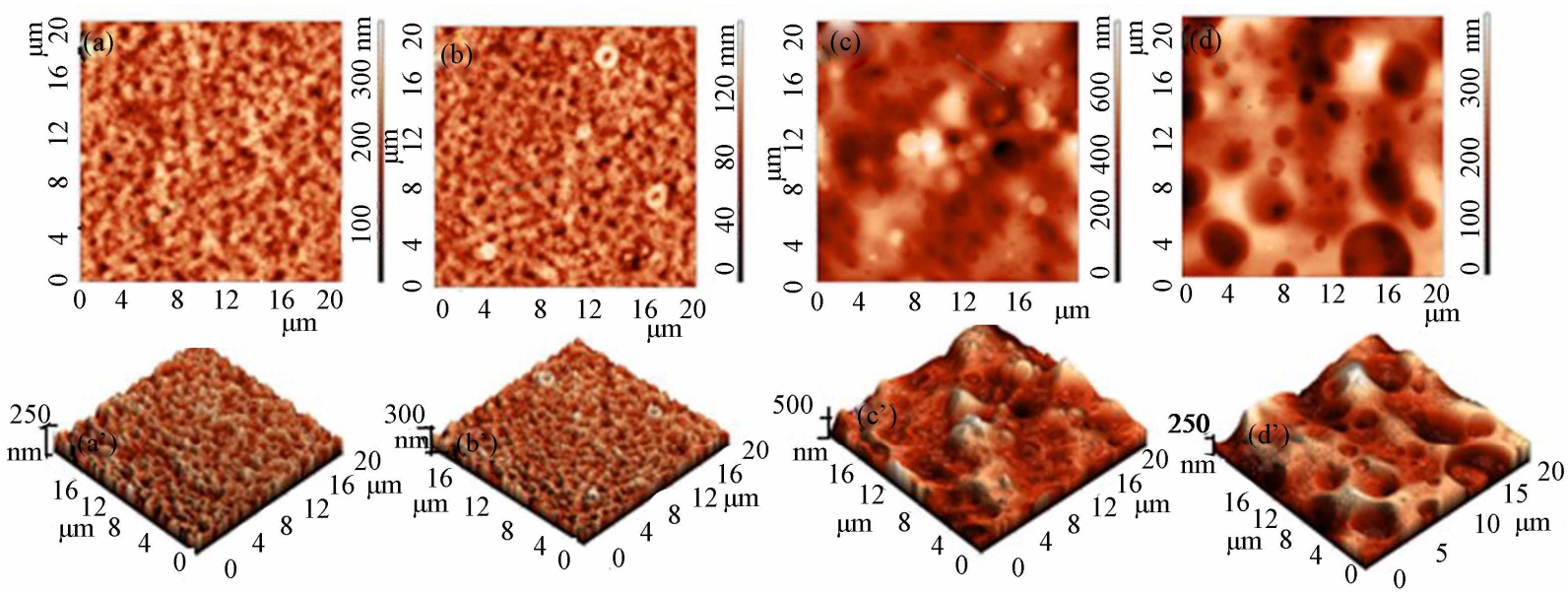

Figure 3. 2D and 3D AFM images with $20 \times 20 \mu \mathrm{m}^{2}$ scanned areas of the PSF-DMEA films obtained from DMF/MeOH solutions: (a, a') - 100/0; (b, b') - 75/25 v/v; (c, c') - 50/50 v/v; (d, d') - 25/75.
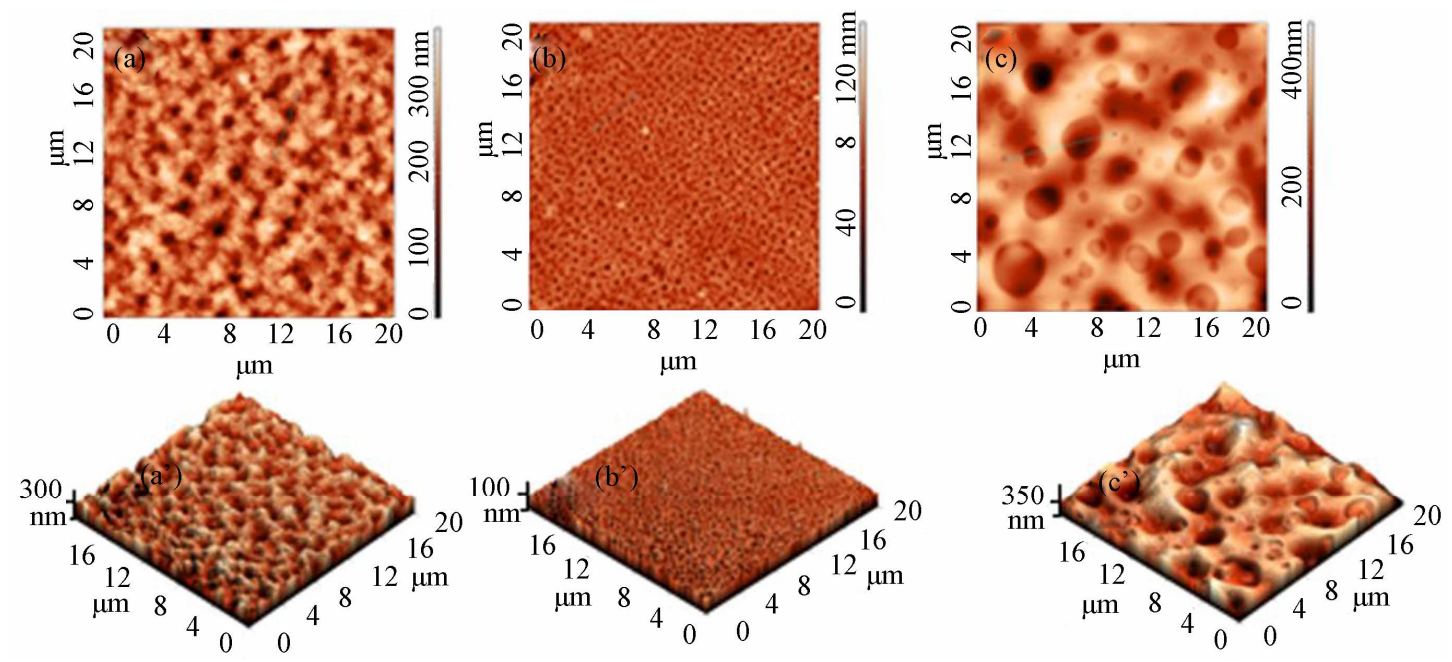

Figure 4. 2D and 3D AFM images with $20 \times 20 \boldsymbol{\mu m}^{2}$ scanned areas of the PSF-DMEA films obtained from DMF/water solutions: (a, a') - 75/25 v/v; (b, b') - 50/50 v/v; (c, c') - 40/60.
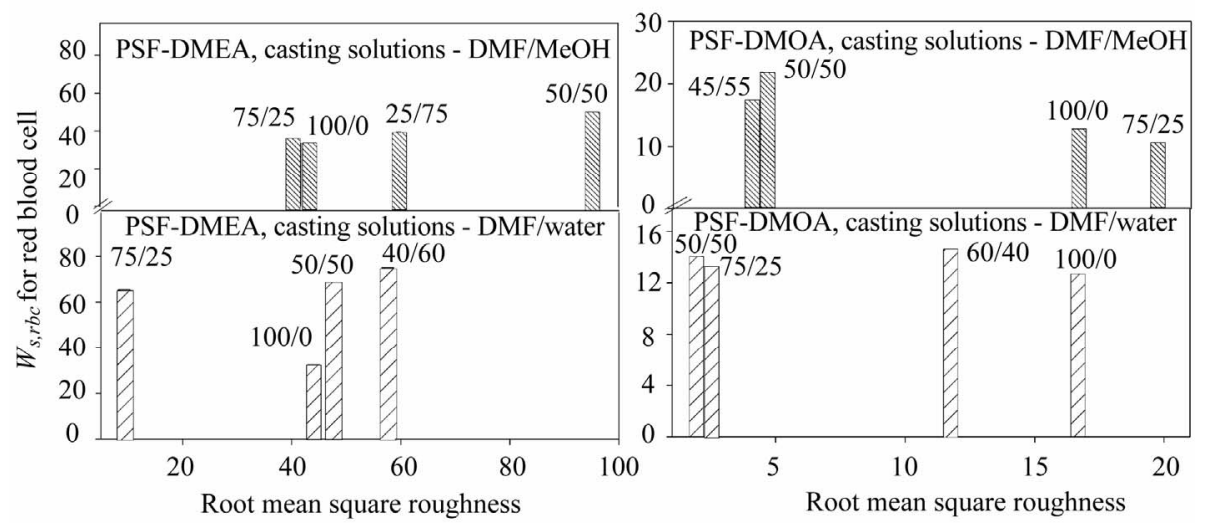

Figure 5. Effect of surface roughness on the work of spreading of red blood cells over the surface of PSF-DMEA and PSF-DMOA films prepared from solutions in different DMF/MeOH and DMF/water solvent mixtures. 
Table 5. Pore characteristics, including number of pores, area $\left(\mu \mathrm{m}^{2}\right)$, depth $(\mathrm{nm})$, diameter $(\mu \mathrm{m})$, length $(\mu \mathrm{m})$, and mean width $(\mu \mathrm{m})$, and surface roughness paramaters, including average roughness $(S a, \mathrm{~nm})$, root mean square roughness $(S q, \mathrm{~nm})$, and average height from the height histogram $(\mathrm{Ha}, \mathrm{nm})$ of PSF-DMEA and PSF-DMOA films prepared from solutions in $\mathrm{DMF} / \mathrm{MeOH}$ and DMF/water $(\% \mathrm{v} / \mathrm{v})$, with $20 \times 20 \mathrm{\mu m}^{2}$ scanned areas, corresponding to the $2 \mathrm{D}$ AFM images.

\begin{tabular}{|c|c|c|c|c|c|c|c|c|c|}
\hline \multirow{2}{*}{ Solvent mixtures } & \multicolumn{6}{|c|}{ Pore characteristics } & \multicolumn{3}{|c|}{ Surface roughness } \\
\hline & Number of pores & Area & Depth & Diameter & Length & Mean width & Sa & $S q$ & $\mathrm{Ha}$ \\
\hline \multicolumn{10}{|c|}{ PSF-DMEA, DMF/MeOH (\% v/v) } \\
\hline $100 / 0$ & 234 & 0.24 & 272.78 & 0.57 & 0.86 & 0.31 & 33.97 & 42.87 & 231.14 \\
\hline $75 / 25$ & 268 & 0.27 & 259.59 & 0.62 & 0.94 & 0.32 & 31.89 & 41.47 & 217.26 \\
\hline $50 / 50$ & 52 & 0.47 & 348.25 & 0.78 & 1.09 & 0.47 & 74.09 & 95.12 & 402.61 \\
\hline $25 / 75$ & 37 & 1.10 & 242.07 & 1.18 & 1.73 & 0.62 & 48.36 & 59.80 & 193.33 \\
\hline \multicolumn{10}{|c|}{ PSF-DMEA, DMF/water (\% v/v) } \\
\hline $75 / 25$ & 147 & 0.51 & 245.19 & 0.86 & 1.25 & 0.48 & 37.41 & 47.76 & 227.79 \\
\hline $50 / 50$ & 1080 & 0.06 & 89.57 & 0.23 & 0.47 & 0.15 & 6.87 & 9.27 & 77.43 \\
\hline $40 / 60$ & 42 & 2.19 & 230.10 & 1.64 & 2.43 & 0.86 & 44.98 & 57.61 & 281.80 \\
\hline \multicolumn{10}{|c|}{ PSF-DMOA, DMF/MeOH (\% v/v) [27] } \\
\hline $100 / 0$ & 9 & 10.43 & 25.64 & 3.33 & 6.24 & 1.45 & 14.11 & 16.82 & 58 \\
\hline $75 / 25$ & - & - & - & - & - & - & 14.43 & 19.90 & 82 \\
\hline $50 / 50$ & 34 & 0.70 & 11.37 & 0.89 & 1.56 & 0.42 & 3.09 & 4.41 & 17 \\
\hline $45 / 55$ & 44 & 0.80 & 16.02 & 0.98 & 1.53 & 0.49 & 2.77 & 4.24 & 28 \\
\hline \multicolumn{10}{|c|}{ PSF-DMOA, DMF/water (\% v/v) [27] } \\
\hline $75 / 25$ & 5 & 3.12 & 13.65 & 1.97 & 3.26 & 0.96 & 1.59 & 2.34 & 15 \\
\hline $60 / 40$ & 27 & 0.04 & 19.54 & 0.22 & 0.35 & 0.11 & 9.19 & 11.83 & 70 \\
\hline $50 / 50$ & 18 & 2.09 & 5.55 & 1.46 & 2.63 & 0.64 & 1.52 & 2.17 & 8 \\
\hline
\end{tabular}

\subsection{Surface Morphology}

It is generally agreed that the physicochemical properties of substratum surfaces are the main factors mediating the compatibility with blood.

Figures 3 and $\mathbf{4}$ plot the bi- and three-dimensional structure evidenced by AFM investigations of PSFDMEA films prepared with 100/0 v/v, 75/25 v/v, 50/50 $\mathrm{v} / \mathrm{v}$ and 25/75 v/v, and also with 75/25 v/v, 50/50 v/v and 40/60 v/v of DMF/MeOH and DMF/water compositions solvent mixtures, respectively. According to the images, increasing the nonsolvent content in the casting solutions favored modification of surface morphology. Thus, Figure 3 and Table 5 show that average surface roughness attains its maximum value at 50/50 v/v DMF/MeOH, and favors the appearance of the smallest number of pores with highest depth values. Also, the area, diameter, length and mean width increase with increasing the nonsolvent content. It should be noted that the thermodynamic quality of the solvent mixtures over the studied domain increases with the addition of nonsolvent, at approximately 50/50 v/v DMF/MeOH becoming constant, while the preferential adsorption of nonsolvent takes a maxim value, according to Figure 1.

The presence of water as a nonsolvent in the solutions used for casting films influenced the AFM images pre- sented in Figure 4; a higher water content decreases the thermodynamic quality of the DMF/water solvent mixtures (Figure 1) so that, at 50/50 v/v DMF/water, a minimum value of surface roughness and a maximum number of pores with minimum values of area, depth, diameter, length and mean width, were observed. It may be assumed that the specific interactions with the mixed solvents employed in the study modify the PSF-DMEA and PSF-DMOA solubility and determine modification of the solution properties [23], according to Figure 1.

On the other hand, previous data [27] obtained for PSF-DMOA in the same solvent mixtures evidenced that the number of pores and their average size increases, while the average surface roughness decreases with increasing the content of nonsolvent, $\mathrm{MeOH}$. For films prepared from DMF/water solutions, the presence of water as a nonsolvent in the casting solution decreases the thermodynamic quality of the DMF/water solvent mixtures up to a $40 \%$ water composition, so that, for the corresponding film, average surface roughness, the number of pores and their depths take maximum values with a minimum area.

Figure 5 plots the dependencies between root mean square roughnesses and the work of spreading of the red blood cells over the surface of PSF-DMEA and PSFDMOA films prepared from different $\mathrm{DMF} / \mathrm{MeOH}$ and 
DMF/water solvent mixtures. These results show that surface morphology depends on the history of the formed films, including the characteristics of quaternized polysulfones and the thermodynamic quality of solvents. Moreover, the results suggest that surface free energy (surface hydrophobicity) and surface roughness are the key parameters controlling the compatibility with the red blood cells, known as a complicated process that depends on many factors, including surface chemistry, hydrophobicity, and surface roughness. The contribution of each of these factors is difficult to establish, however, it is clearly seen that PSF-DMOA is characterized by a lower compatibility with the red blood cells than PSF-DMEA. On the other hand, the compatibility values are higher for PSFDMEA and lower for PSF-DMOA films prepared in DMF/ water, compared with films prepared in DMF/ $\mathrm{MeOH}$.

\section{Conclusions}

New quaternized polysulfones, prepared by quaternization of chloromethylated polysulfone with N,N-dimethylethylamine and $\mathrm{N}, \mathrm{N}$-dimethyloctylamine were investigated to obtain information on their hydrophilic/hydrophobic properties and blood compatibility. The history of the formed films, prepared by a dry-cast process in DMF/ $\mathrm{MeOH}$ and DMF/water solvent/nonsolvent mixtures, influenced the surface tension parameters, surface and interfacial free energy and the work of spreading of water, maintaining the surfaces hydrophobic characteristics of both polysulfones. On the other hand, the results reflect the capacity of N-dimethylethylammonium or N-dimethyloctylammonium chloride pendant groups to determine the acceptor or donor character of the polar terms, caused by the inductive phenomena of alkyl radicals.

The AFM images showed that surface morphology is characterized by roughness and nodules formations, depending on the composition of solvent/nonsolvent mixtures, including the characteristics of quaternized polysulfones and the thermodynamic quality of the solvents. Moreover, the results suggest that:

- $\quad$ surface hydrophobicity and surface roughness are the parameters controlling the compatibility with the red blood cells and platelets: a good hydrophobicity can be correlated with a good adhesion of the red blood cells and with a good cohesions of the platelets on the surface of the quaternized polysulfone films;

- $\quad$ high work of adhesion comparatively with work of cohesion for the red blood cells, but a smaller work of adhesion comparatively with the one of cohesion for platelets was obtained;

- the exposure of blood to both quaternized polysulfones involves higher platelets cohesion for PSFDMOA films, comparatively with PSF-DMEA films.
- $\quad$ These results are useful in investigations on specific biomedical applications, including evaluation of bacterial adhesion to the surfaces, and utilization of modified polysulfones as semipermeable membranes.

\section{REFERENCES}

[1] M. Barikani and S. Mehdipour-Ataei, "Synthesis, Characterization and Thermal Properties of Novel Arylene Sulfone Ether Polyimides and Polyamides," Journal of Polymers Science, Part A: Polymer Chemistry, Vol. 38, No. 9, May 2000, pp. 1487-1492.

doi:10.1002/(SICI)1099-0518(20000501)38:9<1487:AID -POLA11>3.0.CO;2-U

[2] B. K. Mann and J. L West, "Tissue Engineering in the Cardiovascular System: Progress toward a Tissue Engineered Heart,” The Anatomical Record, Vol. 263, No. 4, August 2001, pp. 367-371. doi: 10.1002/ar.1116

[3] A. Higuchi, N. Iwata, M. Tsubaki and T. Nakagawa, "Surface-Modified Polysulfone Hollow Fibers," Journal of Applied Polymer Science, Vol. 36, No. 8, October 1988, pp. 1753-1767. doi: 10.1002/app.1988.070360804

[4] R. N. Johnson, "Polysulfones. Plastics, Resins, Rubbers, Fibers," Encyclopedia of Polymer Science and Technology, Vol. 11, F. M. Herman, G. G. Norman and M. N. Bikales Eds., John Wiley \& Sons, New York, London, Sydney and Toronto, 1969, pp. 447-463.

[5] G. Khang, H. B. Lee and J. B. Park, "Biocompatibility of Polysulfone. I. Surface Modifications and Characterizations," Bio-Medical Materials and Engineering, Vol. 5, No. 4, January 1995, pp. 245-258.

[6] A. Higuchi, K. Shirano, M. Harashima, B. O. Yoon, M. Hara, M. Hattori and K. Imamura, "Chemically Modified Polysulfone Hollow Fibers with Vinylpyrrolidone Having Improved Blood Compatibility,” Biomaterials, Vol. 23, No.13, July 2002, pp. 2659-2666. doi: 10.1016/S0142-9612(01)00406-9

[7] M. Tomaszewska, A. Jarosiewicz and K. Karakulski, "Physical and Chemical Characteristics of Polymer Coatings in CRF Formulation,” Desalination, Vol. 146, No. 1, September 2002, pp. 319-323. doi: 10.1016/S0011-9164(02)00501-5

[8] R. Guan, H. Zou, D. Lu, C. Gong and Y. Liu, "Polyetheresulfone Sulfonated by Chlorosulfonic Acid and its Membrane Characteristics,” European Polymer Journal, Vol. 41, No. 7, July 2005, pp. 1554-1560.

doi: 10.1016/j.europolyml.2005.01.018

[9] H. Yu, Y. Huang, H. Ying and C. Xiao, "Preparation and Characterization of a Quaternary Ammonium Derivative of Konjac Glucomannan,” Carbohydrate Polymers, Vol. 69, No. 1, May 2007, pp. 29-40. doi: 10.1016/j.carbpol.2006.08.024

[10] A. Filimon, E. Avram, S. Dunca, I. Stoica and S. Ioan, "Surface Properties and Antibacterial Activity of Quaternized Polysulfones,” Journal of Applied Polymer Science, Vol. 112, No. 3, May 2009, pp. 1808-1816.

doi: 10.1002/app.29591 
[11] A. Filimon, E. Avram and S. Ioan, "Influence of Mixted Solvents and Temperature on the Solution Properties of Quaternized Polysulfones,” Journal of Macromolecular Science, Part B: Physics, Vol. 46, No. 3, May 2007, pp. 503-520. doi: 10.1080/00222340701257752

[12] S. Ioan, A. Filimon and E. Avram, "Influence of the Degree of Substitution on the Solution Properties of Chloromethylated Polysulfone," Journal of Applied Poly- mer Science, Vol. 101, No. 1, April 2006, pp. 524-531. doi: 10.1002/app.23340

[13] A. Idrisa, N. M. Zaina and M. Y. Noordinb, "Synthesis, Characterization and Performance of Asymmetric Polyethersulfone (PES) Ultrafiltration Membranes with Polyethylene Glycol of Different Molecular Weights as Additives,” Desalination, Vol. 207, No. 1-3, March 2007, pp. 324-339. doi: 10.1016/j.desal.2006.08.008

[14] V. Kochkodan, S. Tsarenko, N. Potapchenko, V. Kosinova and V. Goncharuk, "Adhesion of Microorganisms to Polymer Membranes: A Photobactericidal Effect of Surface Treatment with $\mathrm{TiO}_{2}$," Desalination, Vol. 220, No. 1-3, March 2008, pp. 380-385.

doi: 10.1016/j.desal.2007.01.042

[15] M. D. Guiver, P. Black, C. M. Tam and Y. Deslandes, "Functionalized Polysulfone Membranes by Heterogeneous Lithiation,” Journal of Applied Polymer Science, Vol. 48, No. 9, June 1993, pp.1597-1606. doi: 10.1002/app.1993.070480912

[16] E. Avram, E. Butuc, C. Luca and I. Druta, "Polymers with Pendent Functional Groups. III. Polysulfone Containing Viologen Group," Journal of Macromolecular Science, Part A: Pure Applied Chemistry, Vol. 34, No. 9, September 1997, pp. 1701-1714. doi: 10.1080/10601329708010036

[17] E. Avram, "Polymers with Pendent Functional Groups. VI. A Comparative Study on the Chloromethylation of Linear Polystyrene and Polysulfone with Paraphormaldehyde/Me $\mathrm{MSiCl}_{3}$," Polymer-Plastics Technology and Engineering, Vol. 40, No. 3, May 2001, pp. 275-281. doi: 10.1081/PPT-100000248

[18] C. Luca, E. Avram and I. Petrariu, "Quaternary Ammonium Polyelectrolytes. V. Amination Studies of Chloromethylated Polystyrene with N,N-Dimethylalkylamines," Journal of Macromolecular Science, Part A: Pure Applied Chemistry, Vol. 25, No. 4, 1988, pp. 345-361. doi: 10.1080/00222338808053373

[19] L. Ghimici and E. Avram, "Viscosimetric Behavior of Quaternized Polysulfones," Journal of Applied Polymer Science, Vol. 90, No. 2, October 2003, pp. 465-469. doi: 10.1002/app.12677

[20] S. Ioan, A. Filimon and E. Avram, "Conformation and Viscometric Behavior of Quaternized Polysulfone in Dilute Solution,” Polymer Engineering and Science, Vol. 46, No. 7, July 2006, pp. 827-836. doi: 10.1002/pen.20526

[21] S. Ioan, A. Filimon and E. Avram, "Influence of Substitution Degree to the Optical Properties of Chloromethylated Polysulfone,” Journal of Macromolecular Science, Part B: Physics, Vol. 44, No.1, February 2005, pp. 129-135. doi: 10.1081/MB-200044623
[22] S. Ioan, A. Filimon, E. Avram and G. Ioanid, "Effect of Chemical Structure and Plasma Treatment on the Surface Properties of Polysulfones," e-Polymers, No. 031, March 2007, pp. 1-13 (ISSN 1618-7229).

[23] A. Filimon, R. M. Albu, E. Avram and S. Ioan, "Effect of Alkyl Side Chain on the Conformational Properties of Polysulfones with Quaternary Groups,” Journal of Macromolecular Science, Part B: Physics, Vol. 49, No. 1, January 2010, pp. 207-217. doi: 10.1080/00222340903346494

[24] X. J. Huang, Z. K. Xu, L. S. Wan, Z. G. Wang and J. L. Wang, "Novel Acrylonitrile-Based Copolymers Containing Phospholipid Moities: Synthesis and Characterization,” Macromolecular Bioscience, Vol. 5, No. 4, April 2005, pp. 322-330. doi: 10.1002/mabi.200400165

[25] M. H. Stenzel, C. Barner-Kowollik, T. P. Davis and H. M. Dalton, “Amphiphilic Block Copolymers Based on Poly (2-Acryloyloxyethyl Phosphorylcholine) Prepared via RAFT Polymerisation as Biocompatible Nanocontainers," Macromolecular Bioscience, Vol. 4, No. 4, April 2004, pp. 445-453. doi: 10.1002/mabi.200300113

[26] L. Lewis, J. Berwick, M. C. Davies, C. J. Roberts, J. H. Wang, S. Small, A. Dunn, V. O’Byrne, R. P. Redman and S. A. Jones, "Synthesis and Characterisation of Cationically Modified Phospholipid Polymers,” Biomaterials, Vol. 25, No. 15, July 2004, pp. 3099-3108. doi: 10.1016/j.biomaterials.2003.09.082

[27] S. Ioan, R. M. Albu, E. Avram, I. Stoica and E. G. Ioanid, "Surface Characterization of Quaternized Polysulfone Films and Biocompatiblity Studies," Journal of Applied Polymer Science, Vol. 121, No. 1, July 2011, pp. 127137. doi: 10.1002/app.33380

[28] K. Owens and R. C. Wendt, "Estimation of the Surface Free Energy of Polymers," Journal of Applied Polymer Science, Vol. 13, No. 8, August 1969, pp. 1741-1747. doi: 10.1002/app.1969.070130815

[29] H. Kälble, "Peel Adhesion: Influence of Surface Energies and Adhesive Rheology," Journal Adheshion, Vol. 1, No. 2, April 1969, pp. 102-123.

[30] C. J. van Oss, R. J. Good and M. K. Chaudhury, “Additive and Nonadditive Surface Tension Components and the Interpretation of Contact Angles,” Langmuir, Vol. 4, No. 4, July 1988, pp. 884-891. doi: 10.1021/la00082a018

[31] C. J. van Oss, L. Ju, M. K. Chaudhury and R. J. Good, "Interfacial Lifshitz-van der Waals and Polar Interactions in Macroscopic Systems,” Chemical Reviews, Vol. 88, No. 6, September 1988, pp. 927-941. doi: 10.1021/cr00088a006

[32] M. Rankl, S. Laib and S. Seeger, "Surface Tension Properties of Surface-Coatings for Application in Biodiagnostics Determined by Contact Angle Measurements," Colloids and Surface B: Biointerfaces, Vol. 30, No. 3, July 2003, pp. 177-186. doi: 10.1016/S0927-7765(03)00085-7

[33] R. S. Faibish, W. Yoshida and Y. Cohen, "Contact Angle Study on Polymer-Grafted Silicon Wafers," Journal of Colloid Interface Science, Vol. 256, No. 2, December 
2002, pp. 341-350. doi: 10.1006/jcis.2002.8612

[34] C. J. van Oss, "Interfacial Forces in Aqueous Media," Marcel Dekker, New York, 1994.

[35] K. Vijayanand, K. Deepak, D. K. Pattanayak, T. R. Rama Mohan and R. Banerjee, "Interpenetring Blood-Biomaterial Interactions from Surface Free Energy and Work of Adhesion,” Trends in Biomaterials and Artificial Organs,
Vol. 18, No. 2, January 2005, pp. 73-83.

[36] S. Reitsma, D. W. Slaaf, H. Vink, M. A. M. J. van Zandvoort and M. G. A. oude Egbrink, "The Endothelial Glycocalyx: Composition, Functions, and Visualization," Pflügers Archiv-European Journal of Physiology, Vol. 454, No. 3, June 2007, pp. 345-359. doi: $10.1007 / \mathrm{s} 00424-007-0212-8$ 